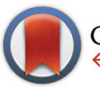

CrossMark \&lick for updates

Cite this: Polym. Chem., 2016, 7, 1234

Received 10th December 2015, Accepted 4th January 2016

DOI: $10.1039 /$ c5py01969a

www.rsc.org/polymers

\section{Ultra-large sheet formation by 1D to 2D hierarchical self-assembly of a "rod-coil" graft copolymer with a polyphenylene backbone $\uparrow$}

\author{
Yinjuan Huang, $t^{\mathrm{a}}$ Rui Yuan, $\stackrel{+}{\mathrm{a}}^{\mathrm{a}}$ Fugui $\mathrm{Xu},{ }^{\mathrm{a}}$ Yiyong Mai, ${ }^{\mathrm{a}}$ Xinliang Feng ${ }^{\mathrm{a}, \mathrm{b}}$ and \\ Deyue Yan ${ }^{a}$
}

\begin{abstract}
This communication reports a unique ultra-large sheet formation through hierarchical self-assembly of a rod-coil graft copolymer containing a rigid polyphenylene backbone and flexible poly(ethylene oxide) (PEO) side chains. The hierarchical self-assembly process involved a distinctive morphological transition of 1D helical to 2D superstructures. The graft copolymer offers a new chance for the challenging bottom-up fabrication of ultra-large self-assembled nanosheets in solution, as well as a novel system for fundamental studies on 2D self-assembly of polymers.
\end{abstract}

"Rod-coil" graft copolymers are an important type of polymer containing a rigid backbone with densely tethered flexible polymeric chains. ${ }^{1-3}$ Their interesting one-dimensional (1D) brush-like structure leads to numerous potential applications, such as molecular actuators, templates for metal nanowires, etc. ${ }^{1,3}$ Conjugated polymers, e.g. polyphenylene and polythiophene, are the major category of rigid backbones in rod-coil graft copolymers, as introducing pendant moieties onto conjugated polymers to form graft copolymers is a general pathway to improve their solubility and tailor their optoelectronic properties. $^{4-6}$

Self-assembly of rod-coil graft copolymers with conjugated polymer backbones and their linear counterpart, rod-coil block copolymers, has attracted much attention in recent decades, as rod-coil copolymers show distinct self-assembly features compared with flexible (coil-coil) copolymer systems due to the introduction of rigid blocks $;{ }^{6-20}$ moreover, the resultant supermolecular nanostructures of conjugated polymers generally exhibit unique thermal, optical or optoelectronic properties. $^{6-20}$ In particular, the self-assembly of the rod-coil block copolymers has been studied extensively. Several key issues, including aggregate morphologies, morphological

\footnotetext{
${ }^{a}$ School of Chemistry and Chemical Engineering, Shanghai Jiao Tong University, 800 Dongchuan RD, Shanghai 200240, P. R. China.E-mail: mai@sjtu.edu.cn

${ }^{b}$ Department of Chemistry and Food Chemistry, Technische Universität Dresden, Mommsenstrasse 4, 01062 Dresden, Germany

$\dagger$ Electronic supplementary information (ESI) available: Experimental details, ESI figures, calculations, etc. See DOI: 10.1039/c5py01969a

$\$$ These authors contributed equally.
}

control approaches, etc., have been explored..$^{7-16}$ For example, Winnik, Manners and coworkers prepared nanocylinders of controlled lengths by the crystallization-driven self-assembly of poly(3-hexylthiophene)-block-poly-(dimethylsiloxane) rod-coil block copolymers. ${ }^{8}$ Although the self-assembly of rod-coil graft copolymers has also been investigated in recent years, ${ }^{6,17-20}$ it remains much less understood compared with that of rod-coil block copolymers.

In the present study, we synthesized a rod-coil graft copolymer containing a laterally expanded poly-para-phenylene backbone (i.e. poly-para-phenylene with dendritic tetraphenylbenzene substituents) tethered with poly(ethylene oxide) (PEO) side chains. Interestingly, the graft copolymer exhibited hierarchical self-assembly behavior in a $\mathrm{CHCl}_{3}-\mathrm{CH}_{3} \mathrm{OH}$ organic cosolvent at room temperature (Fig. 1). Driven by the methanolphobic interaction of the polyphenylene and the crystallization of the PEO chains, the graft copolymer first self-organized into 1D nanowires, which bundled into 10-60 $\mu \mathrm{m}$ ultralong helices, then evolved to $2 \mathrm{D}$ raft-like nanostructures, and, eventually, to ultra-large multilayered nanosheets with remarkable lateral dimensions of $c a$. $10 \mu \mathrm{m} \times 10 \mu \mathrm{m}$ to $100 \mu \mathrm{m} \times 100 \mu \mathrm{m}$, after the aging of the aggregate solution for $c a .2$ days (Fig. 1). To the best of our knowledge, the bottom-up preparation of such large polymeric sheets by supramolecular self-assembly, without support from a planar interface, has been a severe challenge with only a few successful $\operatorname{cases}^{21,22}$ and has not been achieved for graft copolymers before, as planar polymer assemblies usually rolled or closed and formed tubes or vesicles in solution. ${ }^{23}$ In addition, the unprecedented hierarchical self-assembly process involving $1 \mathrm{D}$ helical to $2 \mathrm{D}$ superstructures provides a significant reference for the $2 \mathrm{D}$ selfassembly of polymers.

The synthesis procedure of the rod-coil graft copolymer is illustrated in Scheme $\mathrm{S} 1 \uparrow$ and the experimental details are described in the ESI. $\dagger$ First, Yamamoto polymerization of a dichloro-substituted oligophenylene monomer produced a laterally expanded poly-para-phenylene modified with $-\mathrm{C}_{10} \mathrm{H}_{20} \mathrm{COOCH}_{3}$ (denoted as PP-COOCH${ }_{3}$ ). Then, PP-COOCH was hydrolyzed to PP-COOH. Afterwards, the esterification of 


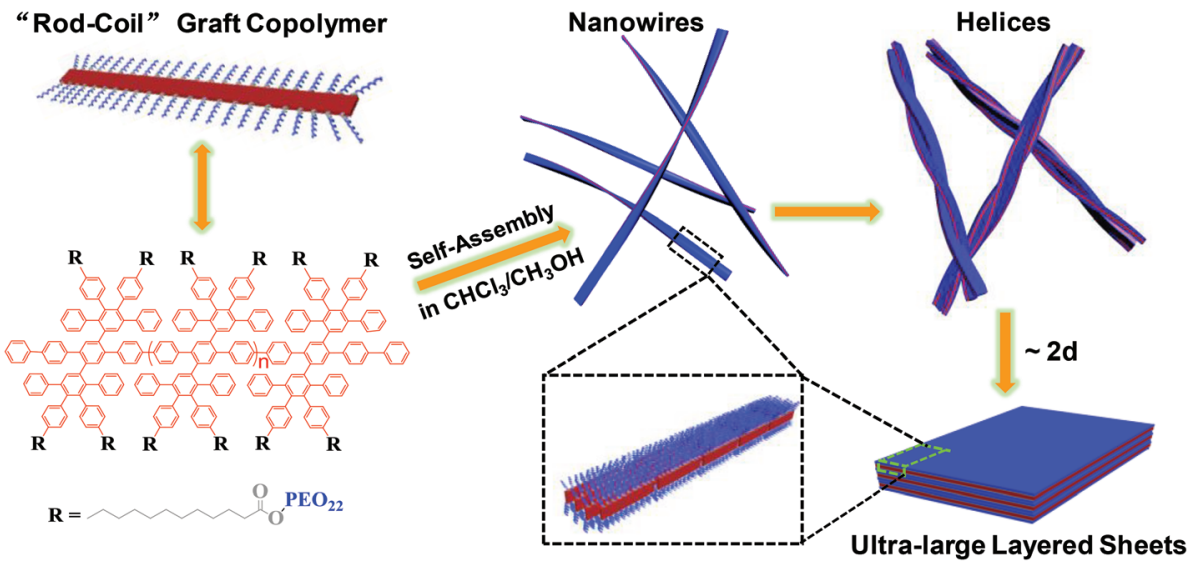

Fig. 1 Schematic illustration of the formation of ultra-large multilayered sheets by 1D to 2D hierarchical self-assembly of the rod-coil graft copolymer at room temperature.

the carboxyl groups on PP-COOH with the hydroxyl groups on $1 \mathrm{~kg} \mathrm{~mol}^{-1}$ poly(ethylene oxide) monomethyl ether yielded the graft copolymer. Fourier transform infrared (FTIR) spectroscopy (Fig. S4A $\dagger$ ) and nuclear magnetic resonance (NMR) (Fig. S5†) measurements validated the successful grafting of the PEO chains.

The grafting percentage (GP) of the copolymer was determined to be $\sim 92 \%$ by NMR, which was supported by the calculation based on elemental analyses (EA) (see pages S8 and S9†). Gel permeation chromatography (GPC) analysis against polystyrene standards in tetrahydrofuran (THF) gave a number-average molecular weight $\left(M_{\mathrm{n}}\right)$ of $136200 \mathrm{~g} \mathrm{~mol}^{-1}$ and a polydispersity index (PDI) of 1.3 for the graft copolymer, as well as a $M_{\mathrm{n}}$ of $51700 \mathrm{~g} \mathrm{~mol}^{-1}$ and a PDI of 1.1 for PP-COOCH (their single-peak distribution GPC curves are presented in Fig. S4B $\dagger$ ). The much larger $M_{\mathrm{n}}$ of the graft copolymer further confirmed the successful grafting of the PEO coils.

The self-assembly of the rod-coil graft copolymer was carried out through a cosolvent method ${ }^{23}$ at room temperature $\left(\sim 20{ }^{\circ} \mathrm{C}\right)$. First, a $\mathrm{CHCl}_{3}$ solution with a concentration of $0.01 \mathrm{mg} \mathrm{mL}^{-1}$ was prepared by dissolving the graft copolymer in $\mathrm{CHCl}_{3}$, which is a common solvent for both polyphenylene and PEO. Then, under gentle stirring, the $\mathrm{CHCl}_{3}$ solution was added dropwise $\left(\sim 60 \mu \mathrm{L} \mathrm{min}{ }^{-1}\right)$ into a 9-fold amount of $\mathrm{CH}_{3} \mathrm{OH}$, a selective solvent for PEO. Finally, a light blue mixed solution was obtained, suggesting the formation of polymer aggregates. The as-prepared solution was incubated at room temperature for a period of days.

The aggregation of the graft copolymer in the $\mathrm{CHCl}_{3}-$ $\mathrm{CH}_{3} \mathrm{OH}$ (v/v $\left.1: 9\right)$ cosolvent was confirmed by ultraviolet-visible (UV-vis) and photoluminescence (PL) spectroscopy (Fig. 2). The maximum absorption of the copolymer in $\mathrm{CHCl}_{3}-\mathrm{CH}_{3} \mathrm{OH}$ red-shifted to $\sim 280 \mathrm{~nm}$ compared with $\sim 270 \mathrm{~nm}$ in $\mathrm{CHCl}_{3}$ (Fig. 2A), indicating $\pi-\pi$ interactions associated with the aggregation of the polyphenylene backbones. ${ }^{9}$ The PL spectra showed the distinct quenching of the photoluminescence of the copolymer in $\mathrm{CHCl}_{3}-\mathrm{CH}_{3} \mathrm{OH}$ (Fig. 2B), further confirming polymer aggregation. In addition, the consecutive quenching
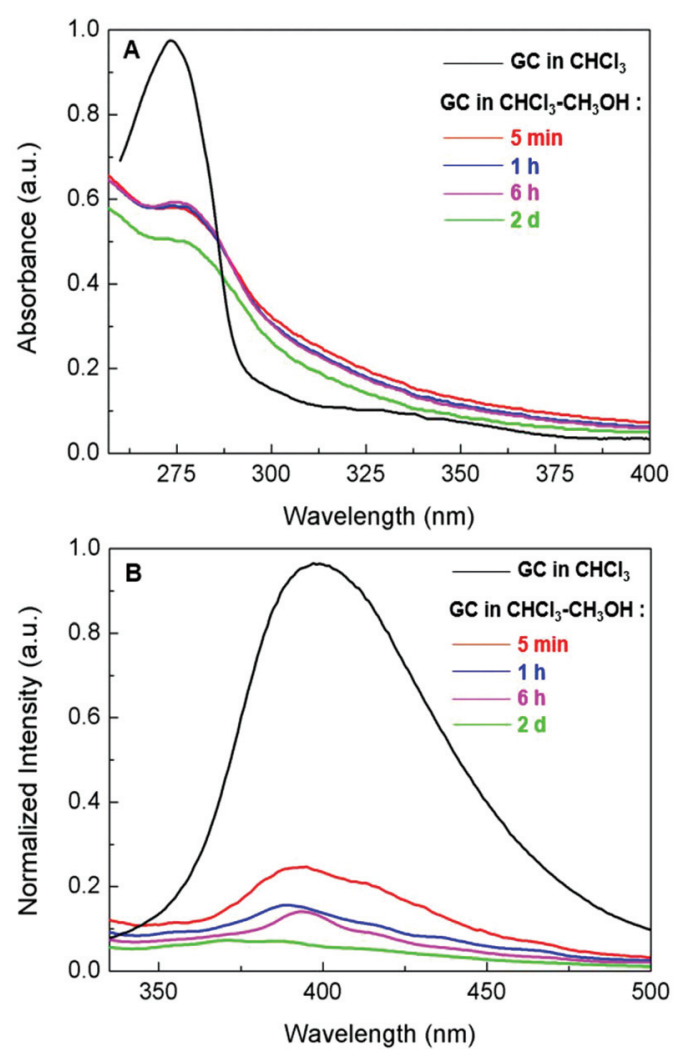

Fig. 2 (A) UV-Vis and (B) photoluminescence spectra of the graft copolymer (GC) in $\mathrm{CHCl}_{3}$ and in $\mathrm{CHCl}_{3}-\mathrm{CH}_{3} \mathrm{OH}$ (v/v 1:9) with the aging (from 5 minutes to 2 days) of the aggregate solution at room temperature (concentration: $10^{-3} \mathrm{mg} \mathrm{mL}^{-1}$ ).

of the photoluminescence with the aging of the aggregate solution over 2 days suggested possible progressive changes in the morphology of the polymer aggregates.

The structures of the polymer aggregates formed in the $\mathrm{CHCl}_{3}-\mathrm{CH}_{3} \mathrm{OH}(\mathrm{v} / \mathrm{v}$ 1:9) solution were examined by transmission electron microscopy (TEM) and atomic force 


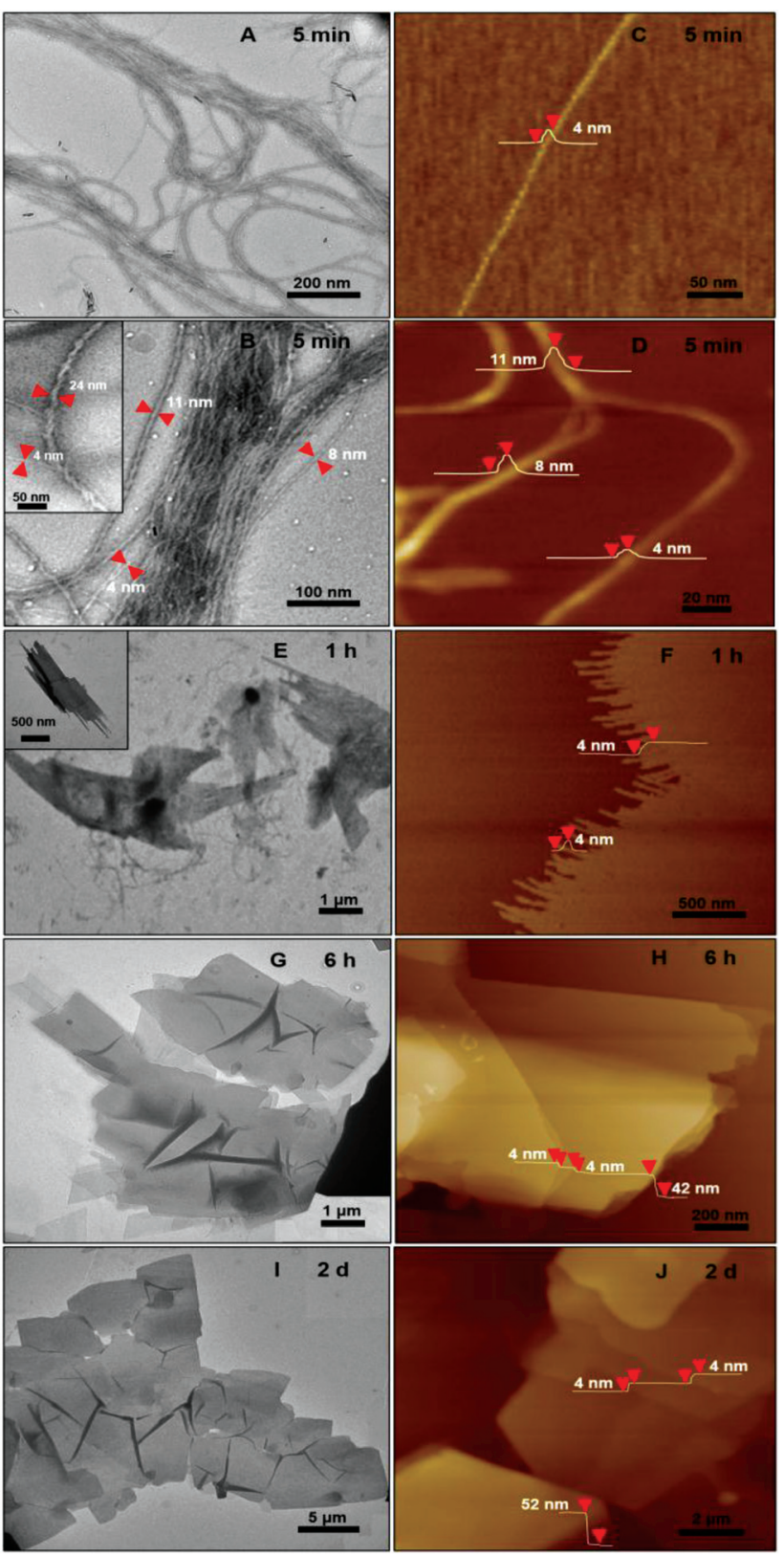

Fig. 3 TEM and AFM images of the assemblies formed by the hierarchical self-assembly of the graft copolymer in the $\mathrm{CHCl}_{3}-\mathrm{CH}_{3} \mathrm{OH}$ (v/v 1:9) solution after 5 minutes (A-D), 1 hours $(E, F), 6$ hours $(G, H), 2$ days $(I, J)$. The relevant dimensions of the assemblies are indicated in the corresponding TEM and AFM images.

microscopy (AFM) (Fig. 3). TEM images revealed a 1D helical structure for the as-formed graft copolymer assemblies in $\mathrm{CHCl}_{3}-\mathrm{CH}_{3} \mathrm{OH}$ (Fig. 3A, B and Fig. S6A, B $\dagger$ ). Left- and righthanded helices coexisted as no chiral moieties were employed during the self-assembly. The size statistics gave an average diameter of $17 \pm 9 \mathrm{~nm}$ and lengths of $10-60 \mu \mathrm{m}$ for the helices. The helices were formed by the twist of 1D aggregates of $\sim 4 \mathrm{~nm}$ width (Fig. 3B and the inset shows a typical highresolution TEM image). The AFM profiles gave a thickness of $\sim 4 \mathrm{~nm}$ for the 1D aggregates and confirmed that they bundled into the helices (Fig. 3C and D). The combination of the $4 \mathrm{~nm}$ width and thickness manifests that the 1D aggregates are supermolecular nanowires. Since the thickness of the nanowires is close to the calculated width $(\sim 3.9 \mathrm{~nm})$ of a graft copolymer molecule while their width is much smaller than the length ( 36 nm) of the copolymer molecules (see the calculation in section 4.3.1 in page $\mathrm{S} 12, \mathrm{ESI} \dagger$ ), it is reasonable to speculate that the nanowires are assembled by an "end-to-end" and "side-by-side" combined alignment of the brush-like molecules, as illustrated in the middle of Fig. 1. The highmagnification TEM images (e.g. Fig. 3B) revealed that most of the helices ( $c a .80 \%$ ) were double- and triple-stranded in terms of their diameters and they coexisted with a number of multistranded helices ( $\geq 4$ strands).

The formation of the helices was found to be kinetically controlled and they were thermodynamically unstable, namely their morphology was quenched temporarily in the mixed solvent with a large amount of $\mathrm{CH}_{3} \mathrm{OH}$ and also in pure methanol after the dialysis of the $\mathrm{CHCl}_{3}-\mathrm{CH}_{3} \mathrm{OH}$ solution. After onehour aging, the helices evolved into "rafts" that consisted of 1D nanostructures (Fig. 3E and F). The thickness of the rafts was $\sim 4 \mathrm{~nm}$ (Fig. 3F), the same as that of the aforementioned nanowires. Therefore, it is believed that the rafts were singlelayered and formed by the planar alignment of the nanowires. The unexpected transformation of $1 \mathrm{D}$ helical to $2 \mathrm{D}$ raft-like superstructures has never been documented before in $2 \mathrm{D}$ supramolecular self-assembly. With the aging of the aggregate solution for $\sim 2$ days, the rafts further developed into ultralarge sheets with an average thickness of $56 \pm 4 \mathrm{~nm}$ and lateral dimensions of $c a .10 \mu \mathrm{m} \times 10 \mu \mathrm{m}$ to $100 \mu \mathrm{m} \times 100 \mu \mathrm{m}$ (Fig. 3G-J and S6D and E†). The greater thickness than that of the single-layered rafts indicated a multilayered structure of the sheets. The wrinkles observed by TEM on the nanosheets reflected a flexible feature (Fig. 3G, I and S6D $\dagger$ ). Afterwards, no big changes were observed on the dimensions of the sheets. The ultra-large sheets suspended in solution were also observed by optical microscopy (Fig. S7†), confirming that the sheets were formed and free-standing in solution. Dynamic light scattering (DLS) measurements revealed an increase in the hydrodynamic diameter of the graft copolymer assemblies with the aging of their solution (Fig. 4 and S8†), confirming the progressive growth of the copolymer assemblies in $\mathrm{CHCl}_{3}-\mathrm{CH}_{3} \mathrm{OH}$.

In light of previous studies on PEO crystallization-driven 2D self-assembly of polymers in solution, ${ }^{24-26}$ we consider that PEO crystallization in organic media contributed to the solution-growth of the ultra-large multilayered sheets. Micro differential scanning calorimetry ( $\mu$ DSC) analysis gave a crystallization temperature $\left(T_{\mathrm{c}}\right)$ of $\sim 26^{\circ} \mathrm{C}$ for the PEO coils in the copolymer aggregates in $\mathrm{CHCl}_{3}-\mathrm{CH}_{3} \mathrm{OH}$ (Fig. S9†). Note that the $2 \mathrm{D}$ self-assembly of the graft copolymer occurred (at $\sim 20{ }^{\circ} \mathrm{C}$ ) below the $T_{\mathrm{c}}$ of the PEO chains. In addition, a short average distance of $\sim 0.7 \mathrm{~nm}$ between neighboring PEO chains on the polyphenylene backbone would be favorable for the crystallization of the PEO coils if the graft copolymer aggregated into sheet-like structures, as the short distance resulted in a high number density of PEO chains at the sheet surfaces, 


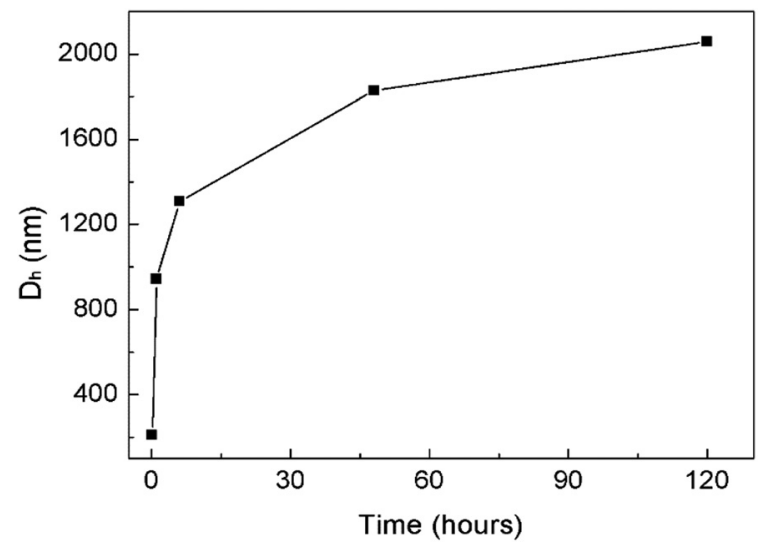

Fig. 4 DLS results showing an increase in the hydrodynamic diameter $\left(D_{\mathrm{h}}\right)$ of the graft copolymer assemblies with the aging of their solution at room temperature. The corresponding DLS plots are presented in Fig. S8. $\dagger$

which exceeded the onset density for the crystallization of the PEO chains below their $T_{\mathrm{c}}$ (see the calculations in section 4.3.2 in the ESI, pages S13 and S14 $\dagger)^{26,27}$ Thus, it is reasonable to believe that the crystallization of the PEO chains acted as a key driving force for the formation of the ultra-large sheets. Two additional lines of evidence also support this point. First, the graft copolymer with a lower GP of $\sim 60 \%$ did not form multilayered sheets under similar experimental conditions, probably due to the larger average distance between adjacent PEO coils on the polyphenylene backbone. Second, at a temperature $\left(\sim 30^{\circ} \mathrm{C}\right)$ higher than the $T_{\mathrm{c}}$ of the PEO chains, the graft copolymer produced irregular aggregates rather than sheets in $\mathrm{CHCl}_{3}-\mathrm{CH}_{3} \mathrm{OH}$.

Based on the above-mentioned results, a possible mechanism for the $1 \mathrm{D}$ to $2 \mathrm{D}$ hierarchical self-assembly of the graft copolymer is illustrated in Fig. 1. In the $\mathrm{CHCl}_{3}-\mathrm{CH}_{3} \mathrm{OH}$ solution, driven by the methanolphobic interaction, the graft copolymer first self-organized into $4 \mathrm{~nm}$ thin nanowires with a polyphenylene layer sandwiched by PEO coils on both up and down sides (middle of Fig. 1). Because of the relatively low PEO number density on the other two sides of the polyphenylene layer, the nanowires tend to associate to minimize the polyphenylene-methanol contact. Around the associated nanowires the PEO coils were crowded, as the estimated radius of gyration $\left(R_{\mathrm{g}}=\sim 1.5 \mathrm{~nm}\right.$ (ref. 28)) of a $1 \mathrm{~kg} \mathrm{~mol}{ }^{-1}$ PEO chain at its end-free state in solution is much larger than the short average distance of $0.7 \mathrm{~nm}$ between neighboring PEO chains in the graft copolymer in the nanowires. Hence, the steric interaction among the PEO chains drove the twist of the associated nanowires to provide more peripheral space for the PEO coils, thus leading to the formation of $1 \mathrm{D}$ helices. At this point, it is worth mentioning that although helix formation represents a self-assembly motif for some chiral polymers, owing to the ordered helical arrangement of their chiral moieties, ${ }^{10,11,15}$ it rarely happens to achiral polymers; ${ }^{9,29,30}$ nevertheless, in the present work, long helices were frequently found in the self-organization of the achiral graft copolymers in the $\mathrm{CHCl}_{3}-\mathrm{CH}_{3} \mathrm{OH}$ cosolvent system, which can be attributed to their unique rod-coil brush-like structure with a short average distance between adjacent PEO chains on the rigid polyphenylene backbone. However, since the helix formation occurred below the $T_{\mathrm{c}}$ of the PEO coils, the crystallization and the resulting compact state of the PEO chains alleviated their steric repulsion and thus favored the transformation of the helices to the thermodynamically more stable sheet-like structures. During the transformation, an untwist process of the helices could be considered for their transition to the singlelayered rafts which further grew to the ultra-large multilayered sheets driven by the PEO crystallization.

Soft nanosheets with lateral dimensions of over $10 \mu \mathrm{m} \times$ $10 \mu \mathrm{m}$ are quite difficult to achieve by the self-assembly of polymers in solution without support from a planar interface; only a few successful cases were documented. ${ }^{21,22}$ In the present work, the rod-coil brush-like structure of the graft copolymer accounts for the ultra-large lateral sizes of the sheets, since the molecules of such an architecture incline to form assemblies with a sandwich structure, in which the laterally expanded poly-para-phenylene rigid backbones locate in the middle layer (Fig. 1). Such a sandwich structure along with the PEO crystallization is in favor of the growth of assemblies in 2D directions. Moreover, the rigid polyphenylene layer and the PEO crystallization can limit the rolling or closing of the sheets, which would generate tubes or vesicles as usually seen in the self-assembly of many flexible polymer systems. ${ }^{23,31,32}$

In summary, this work demonstrates a unique sheet formation by $1 \mathrm{D}$ to $2 \mathrm{D}$ hierarchical self-assembly of a rod-coil graft copolymer comprising a poly-para-phenylene backbone and PEO side chains. Driven by the methanolphobic interaction of the polyphenylene and the crystallization of the PEO chains in a $\mathrm{CHCl}_{3}-\mathrm{CH}_{3} \mathrm{OH}$ mixed organic solvent, the graft copolymer exhibited an unprecedented hierarchical selfassembly process from nanowires to 10-60 $\mu \mathrm{m}$ ultralong helices, then to single-layered "rafts", and finally to ultra-large multilayered nanosheets with lateral dimensions of $c a .10 \mu \mathrm{m}$ $\times 10 \mu \mathrm{m}$ to $100 \mu \mathrm{m} \times 100 \mu \mathrm{m}$. The rod-coil graft polymers afford a new chance for the so far challenging preparation of ultra-large self-assembled nanosheets in solution, as well as a novel system for fundamental studies on 2D self-assembly of conjugated polymers, including the mechanism, factors affecting the morphology, potential applications, etc.

\section{Acknowledgements}

The authors acknowledge the financial support from the 973 Programs of China (2012CB933404 and 2013CBA01602), the Natural Science Foundation of China (21320102006, 21304057 and 51573091), the Natural Science Foundation of Shanghai (13ZR1421200), the Program for Eastern Scholar in Shanghai, and the MPI Partner Group Project for Polymer Chemistry of Graphene Nanoribbons. The authors also appreciate the Instrumental Analysis Center of Shanghai Jiao Tong University for some measurements. 


\section{Notes and references}

1 J. Yuan, Y. Xu and A. H. E. Müller, Chem. Soc. Rev., 2011, 40, 640.

2 J. Rzayev, ACS Macro Lett., 2012, 1, 1146.

3 R. Verduzco, X. Li, S. L. Pesek and G. E. Stein, Chem. Soc. Rev., 2015, 44, 2405.

4 S. S. Sheiko, B. S. Sumerlin and K. Matyjaszewski, Prog. Polym. Sci., 2008, 33, 759.

5 C. Li, M. Liu, N. G. Pschirer, M. Baumgarten and K. Müllen, Chem. Rev., 2010, 110, 6817.

6 Y. Huang, Y. Mai, X. Yang, U. Beser, J. Liu, F. Zhang, D. Yan, K. Müllen and X. Feng, J. Am. Chem. Soc., 2015, 137, 11602.

7 S. A. Jenekhe and X. L. Chen, Science, 1998, 279, 1903.

8 S. K. Patra, R. Ahmed, G. R. Whittell, D. J. Lunn, E. L. Dunphy, M. A. Winnik and I. Manners, J. Am. Chem. Soc., 2011, 133, 8842.

9 E. Lee, B. Hammer, J.-K. Kim, Z. Page, T. Emrick and R. C. Hayward, J. Am. Chem. Soc., 2011, 133, 10390.

10 R.-M. Ho, Y.-W. Chiang, S.-C. Lin and C.-K. Chen, Prog. Polym. Sci., 2011, 36, 376.

11 C. Cai, Y. Li, J. Lin, L. Wang, S. Lin, X.-S. Wang and T. Jiang, Angew. Chem., Int. Ed., 2013, 52, 7732.

12 Y. Zheng, H. Zhou, D. Liu, G. Floudas, M. Wagner, K. Koynov, M. Mezger, H.-J. Butt and T. Ikeda, Angew. Chem., Int. Ed., 2013, 52, 4845.

13 A. Yassar, L. Miozzo, R. Gironda and G. Horowitz, Prog. Polym. Sci., 2013, 38, 791.

14 A. C. Kamps, M. H. M. Cativo, M. Fryd and S.-J. Park, Macromolecules, 2014, 47, 161.

15 J. F. Reuther, D. A. Siriwardane, R. Campos and B. M. Nova, Macromolecules, 2015, 48, 6890.

16 H.-C. Ho, Y.-H. Lee, C.-A. Dai, R. A. Segalman and W.-F. Su, Macromolecules, 2009, 42, 4208; S.-H. Lin, S.-J. Wu, C.-C. Ho and W.-F. Su, Macromolecules, 2013, 46, 2725; C.-C. Ho, S.-J. Wu, S.-H. Lin, S. B. Darling and W.-F. Su, Macromol. Rapid Commun., 2015, 36, 1329.
17 J. H. Yao, K. Y. Mya, L. Shen, B. He, L. Li, Z. Li, Z. Chen, X. Li and K. P. Loh, Macromolecules, 2008, 41, 1438.

18 C. Cai, J. Lin, T. Chen and X. Tian, Langmuir, 2010, 26, 2791.

19 G. M. Miyake, R. A. Weitekamp, V. A. Piunova and R. H. Grubbs, J. Am. Chem. Soc., 2012, 134, 14249.

20 Y. Li, T. Jiang, S. Lin, J. Lin, C. Cai and X. Zhu, Sci. Rep., 2015, 5, 10137.

21 J.-K. Kim, E. Y. Lee, H. Jeong, J.-K. Lee, W.-C. Zin and M. Lee, J. Am. Chem. Soc., 2007, 129, 6082.

22 K. T. Nam, S. A. Shelby, P. H. Choi, A. B. Marciel, R. Chen, L. Tan, T. K. Chu, R. A. Mesch, B.-C. Lee, M. D. Connolly, C. Kisielowski and R. N. Zuckermann, Nat. Mater., 2010, 9, 454.

23 Y. Mai and A. Eisenberg, Chem. Soc. Rev., 2012, 41, 5969.

24 G. Rizis, T. G. M. van de Ven and A. Eisenberg, Angew. Chem., Int. Ed., 2014, 53, 9000.

25 J. R. Sun, X. S. Chen, C. L. He and X. B. Jing, Macromolecules, 2006, 39, 3717.

26 R. M. Van Horn, J. X. Zheng, H.-J. Sun, M.-S. Hsiao, W.-B. Zhang, X.-H. Dong, J. Xu, E. L. Thomas, B. Lotz and S. Z. D. Cheng, Macromolecules, 2010, 43, 6113.

27 J. X. Zheng, H. Xiong, W. Y. Chen, K. Lee, R. M. Van Horn, R. P. Quirk, B. Lotz, E. L. Thomas, A.-C. Shi and S. Z. D. Cheng, Macromolecules, 2006, 39, 641.

28 The $R_{\mathrm{g}}$ for a PEO chain at its end-free state in solution is estimated using $R_{\mathrm{g}}{ }^{2}=b^{2} N_{\mathrm{b}} / 6$ from: M. Rubenstein and R. H. Colby, Polymer Physics, Oxford University Press, New York, 2004, where $b=0.8 \mathrm{~nm}$ for PEO.

29 S. Zhang, H. Cui, Z. Chen, K. L. Wooley and D. J. Pochan, Soft Matter, 2008, 4, 90.

30 J. Dupont, G. Liu, K.-I. Niihara, R. Kimoto and H. Jinnai, Angew. Chem., Int. Ed., 2009, 48, 6144.

31 Y. Zhou and D. Yan, Chem. Commun., 2009, 1172.

32 A. Blanazs, S. P. Armes and A. J. Ryan, Macromol. Rapid Commun., 2009, 30, 267. 